\title{
A MULTI-AGENT SYSTEM FOR SMART COORDINATION OF DYNAMIC SUPPLY CHAINS
}

\author{
Ricardo J. Rabelo; Alexandra A. Pereira-Klen; Edmilson R. Klen \\ Federal University of Santa Catarina, BRAZIL \\ (rabelo; klen; erklen)@gsigma-grucon.ufsc.br
}

This paper describes a multi-agent decision support system called $S C^{2}$ (Supply Chain Smart Coordination) that aims at offering an integrated environment to better manage dynamic supply chains by covering the production, distribution and sales chains. Reliable, timely and interoperable information are exchanged among the supply chain members in XML by means of a workflowdriven and CORBA-based platform, supporting the user in smart decisionmaking. This work has been developed in the scope of the IST DAMASCOS project, focusing on the fashion supply chains of SMEs. The general problem and framework are described, and some results are shown and discussed.

\section{INTRODUCTION}

This paper presents an approach on how a dynamic supply chain (DSC) can be managed in a smart way, focusing on a decision support system developed to assist the enterprises in the coordination of distributed business processes.

Industry nowadays has been crossing a moment where demand is driven more and more by customer and consumer needs. This in turn makes the e-marketplace become an even more independent environment. These trends affect directly the fashion industry in which currently predominating trade and distribution systems are strongly segmented. The Point-of-Sale assesses demand 20 to 40 weeks ahead and orders the goods according to season (Ferreira et al., 00 ). This very long cycle time associated with a demand-driven environment is requiring from system developers an special attention concerning the conception and implementation of an adequate architecture to support the businesses being carried out along the supply chain (SC). Looking at the literacy and the systems available in the market, there are some aspects not very well tackled but that are essential for agile decision-making in a DSC scenario: i) complete view of the entire supply chain so that a better decisionmaking can be taken; (ii) reliable information about the supply chain in terms of current suppliers' capacities, stocks and material flow; (iii) orders follow-up; (iv) commitment with the delivery dates; (v) balanced workload along the supply chain.

By focusing on a Business Intelligence architecture that utilizes common databases and end-user application, the authors believe that it will be possible to address the various information needs of different SC sectors other than fashion. In this sense, this paper stresses the $\mathrm{SC}^{2}$ (Supply Chain Smart Coordination) system, a multi-agent-based decision support system that offers some contribution to those week points mentioned above. It has been developed in the IST DAMASCOS project (Damascos), whose ultimate goal is to develop an open and low cost platform and management services for SMEs involved in DSC of fashion industries.

The original version of this chapter was revised: The copyright line was incorrect. This has been corrected. The Erratum to this chapter is available at DOI: 10.1007/978-0-387-35585-6_68 
The paper is organized as follows: Chapter 2 gives an overview of the difficulty to manage supply chains. Chapter 3 provides a general description of the DAMASCOS and SC $^{2}$ approaches. Chapter 4 shows some implemented results of $\mathrm{SC}^{2}$. Chapter 5 discusses the results achieved.

\section{PROBLEM DESCRIPTION}

One of the main problems to work with DSC is the management of the information of the whole SC. Demand, production capacities, stocks, material flow are some of the information data that should be quickly analyzed to attend an even more and more demanding market. The DAMASCOS project aims at closing the gap between consumer behavior and producer by improved information flow and demand prediction in the sales and distribution networks (Ferreira et al., 00).

A DSC is based on the "traditional" SC where aspects from raw material suppliers up to final consumers - including main producer, distribution centers, sales agents, retailers and so on - are covered. The main difference is that the core partners do not remain the same for a large period of time, and the focus is on the material and product flows as well as on the related business information (Camarinha-Matos et al., 99). Hence, the coordinator of a DSC has the tough task of managing the information and material flows of the distributed business process(es) being carried out by the supply chain and the various relationships between business processes, supply chain members, etc. Figure 1 shows how this task can be complex for a scenario of 10 supply chain members (1 pre-supplier, 4 suppliers, 1 principal producer, 1 sales agent and 3 retailers) and just five end products. The thickest arrows indicate the information flow and the material flow. The fact is that this complexity exponentially increases with the number of supply chain members and end products (and the quantity of its sub-components).



Figure 1-Material and information flow of a Supply Chain 
The $\mathrm{SC}^{2}$ comes to provide a leaner and smart environment where material and information flows of a DSC can be monitored, hence supporting the SC coordinator in managing distributed business processes (Pereira-Klen et al., 01a).

\section{THE DAMASCOS / SC ${ }^{2}$ APPROACH FOR COORDINATION}

Cooperation is a key aspect for the Virtual Organization (VO) paradigm realization and it has been addressed in many different research projects. A cooperation involves sharing information and other resources, as well as communication, enterprise relationships and collaborative activities. Activities carried out by a company are usually organized in clusters of inter-related activities called "process" (business processes - BP), where each BP is designed to achieve an (partial) objective. When properly orchestrated, the combination of various BPs will lead to the achievement of a global VO goal. Therefore, cooperation requires coordination (Camarinha et al., 00). It is desirable that coordination can be accomplished in the most efficient way, then involving flexibility, configuration, and the usage of supporting standards as the composition of the enterprises is dynamic.

In the DAMASCOS project, the coordination model is hierarchical, composed of three levels (Ferreira, et al., 00): Workflow backbone, as a low coordination level; Advanced Coordination Functionalities, as a middle coordination level; Smart Coordination Functionalities, as a high coordination level.

\subsection{The DAMASCOS Suite and the Low Coordination Level}

At the low level, the coordination in the network is supported by a distributed workflow coordination system called Workflow Backbone (WfBB). It provides a lean way to interoperate with the DAMASCOS modules both intra DAMASCOS Suite and inter-enterprises. It is assumed that the "DAMASCOS layer"-composed of a set of modules (a suite) - would be installed on the top of the enterprises' legacy systems so that the enterprises can cooperate with each other.

Besides the WfBB, the DAMASCOS suite (Ferreira, et al., 00) is composed of the following modules (Figure 2): SALSA (for sales support), $\mathrm{D}_{3} \mathrm{~S}_{2}$ (for forecasting purposes), IDLS (support for individual logistics), IPO (support for some production management) and $\mathrm{SC}^{2}$ - the module being addressed in this paper - which offers a support for the management of the entire SC.

In the DAMASCOS suite, each module is in fact represented by a set of "execution functions" put available in its "external interface" (its ORB / CORBA model). For a given business model, every BP is then constituted by a logical composition of a set of cross-modules Business Functions (BFs) - the execution model itself. The execution can be both intra-enterprise, where BFs are executed within a single enterprise, i.e. intra-DAMASCOS suite, and inter-enterprises BFs, when the execution of BFs involves another enterprises in a typical B2B transaction. Therefore, the WfBB will coordinate the execution of every (set of clusters of BFs that compose a) BP.

The information to exchange among the modules is modeled in XML, whose structure respects the involved BFs' information requirements of a given BP. Besides that, in the XML files the "context" of the message is identified, i.e. the type of information it is about. When a module wants to send an information to the other modules, a XML file is published in the WfBB. Internally, the WfBB recognizes the 
context and sends that XML file to the modules previously subscribed to receive it. Finally, via a pooling mechanism, the modules take it for their internal purposes, following the BP global logic, storing the information in a local database.

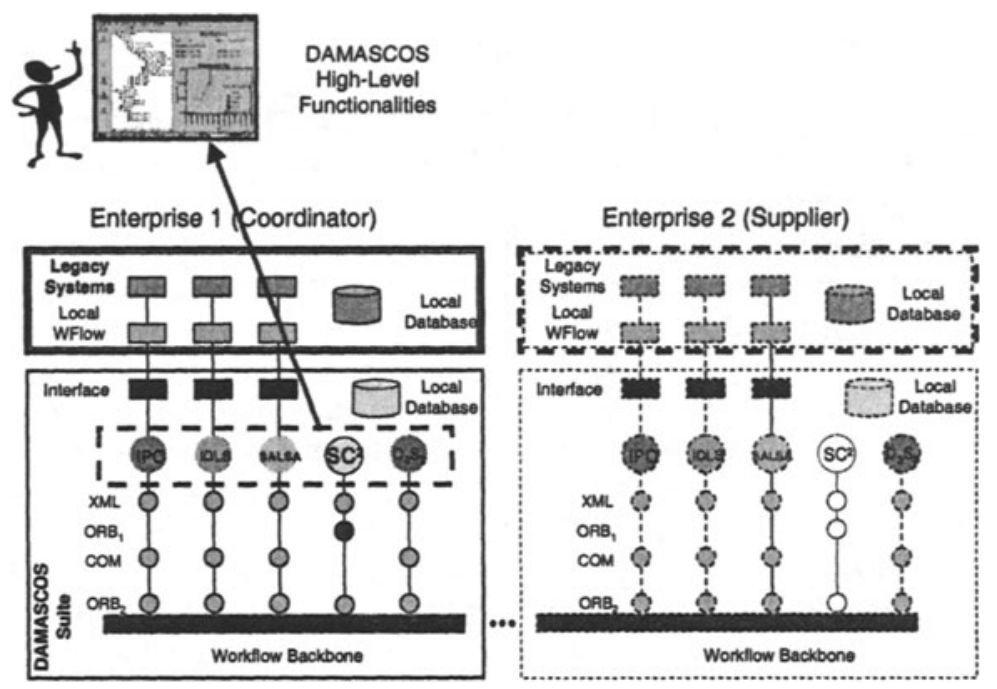

Figure 2 - The DAMASCOS Suite and Framework

\subsection{The Middle and High Coordination Levels}

Whereas the level above focuses on the basic operational side of the BP execution, in DAMASCOS there are two other levels whose focus is on the "intelligence" side (Figure 3). There are many perspectives to exploit the intelligence on how a BP can be managed. In the $\mathrm{SC}^{2}$ approach, a BP execution is tackled via two upper levels of abstraction / coordination: middle and high (Rabelo et al., 00).



Figure 3 - DAMASCOS Coordination Approach

The middle coordination view appears considering that a SC in consumer goods industries typically involves the coordination of small firms at the production, distribution and sales levels. This means to treat a BP from individual parts of the $\mathrm{SC}$ with specific functionalities for each one, called advanced coordination.

In a further stage, taking into account that the chains are normally coordinated and initiated by the consumers goods (principal) producer as the central part of the chain, it is also necessary to extend those functionalities to a higher coordination view, where the smart coordination functionalities are placed. They are "meta" functionalities to manage the dynamic supply network as a whole. 


\section{THE SC ${ }^{2}$ SYSTEM}

The $\mathrm{SC}^{2}$ is a multi-agent-based decision support system that manages the distributed business process of a dynamic supply network by means of real-time monitoring and supervision activities. It facilitates the conflict analysis and its resolution supported by (flexible and configurable) decisional protocols, providing a human-centered smart coordination of the SC.

The whole process starts when the principal producer (the SC coordinator [SCC]) wants to monitor, after a given SC is created, the execution of a particular business process (BP) at a given SC-Member's (SC-M) site. It is supported by means of the sending information from each SC-M to the SC-C (like order status, remaining process time, stock information, etc.) via the WfBB (as described above).

\subsection{The SC ${ }^{2}$ Agents}

$\mathrm{SC}^{2}$ is composed of three classes of agents: smart supervisor, chain supervisors, and supporting agents. The first one is the agent called Smart Agent, responsible for the global SC management. The second one has three instances: Production Agent, Sales Agent and Distribution Agent, responsible for dealing with the production, sales and distribution individual chains, respectively. The third and last one is represented by the XML Agent, which is responsible for dealing with the communication among the agents and with the "external environment" (Figure 4).



Figure $4-\mathrm{SC}^{2}$ Architecture

The $\mathrm{SC}^{2}$ implementation model combines multi-agent systems, CORBA, XML, databases, and decision support systems. As a typical information-consumer module, $S^{2}{ }^{2}$ receives the required information from IPO, IDLS and SALSA modules via the WfBB module, regarding the "contexts". Each of these contexts corresponds to a respective XML DTD, accessed under a pooling philosophy by a $\mathrm{SC}^{2}$ 's ORB specific service. Once the information is got, it is sent and managed by the $X M L$ agent. This agent parsers the XML message received and makes a matching with its internal DTD. After this pre-processing, the information is encapsulated as an object and sent to the $\mathrm{SC}^{2}$ database. The involved DBMS gets this object and stores it in a 
reference data model called Distributed Business Process (DBP) Model (PereiraKlen et al., 00b). In parallel, the XML agent sends a message to the Production, Sales and Distribution agents so that they can be aware about the arrival of updated information. Hence the agents access the information only when they need.

Along a BP operation/execution, the DBP model can be constantly read or updated, both by the XML agent and by the other $\mathrm{SC}^{2}$ agents. Results produced by the agents / SC ${ }^{2}$ are shown to the end-user via graphical interfaces. From the enduser point of view, it is totally transparent the agent being activated to provide some results. (S)he sees the $\mathbf{S C}^{2}$ as a set of "encapsulated" functionalities.

\subsection{Why Multi-agents ?}

The Multi-Agent System (MAS) technology has emerged as a powerful technology to support a cooperative resolution of distributed problems (Jennings, 94). In general, A MAS system corresponds to a network of problem solvers/subsystems agents - that works together to solve problems that are beyond their individual capabilities. In practical terms, in the case of the $\mathrm{SC}^{2}$ system, the main value-added with the adoption of a MAS approach are (Rabelo et al., 01):

- The SC ${ }^{2}$ agents can be launched in distributed PCs;

- The integrated view of the entire supply chain is supported via a cooperative interaction among the $\mathrm{SC}^{2}$ agents, with flexible constraint relaxation;

- The $\mathrm{SC}^{2}$ agents can reason / negotiate about the information exchanged among them and some intelligent deductions (new knowledge) emerge from this;

- Other agents / supply chain perspectives can be added into the $\mathrm{SC}^{2}$ without altering its control architecture;

- Each perspective of the chain is managed in/by an autonomous way/agent.

\subsection{SC ${ }^{2}$ Functionalities}

As the amount of information flowing inside the $\mathrm{SC}$ is enormous, the degree of complexity in managing the information of DSC as well as coordinating its DBPs is very high. The use of information technology is a catalyst to improve the SC performance and is being encouraged for sharing and exchanging information among organizations in different places. That is a new strategy of conducting business (Filos et al., 00). The $\mathrm{SC}^{2}$ follows this trend, where a deep system requirements analysis was made (Pereira-Klen et al., 00). The implemented functionalities in the $\mathrm{SC}^{2}$ are:

- Supply Chain Configurator

Graphical and interactive specification of the "actors" of the SC, comprising their roles and inter-relations. It is the kick-off to activate for the other functionalities.

- Supply Chain Smart Map

This functionality aims to offer to the SC coordinator a graphical and easy-to-use possibility of seeing the multiple production, distribution and sales stages and the main characteristics of each one of these stages. Figure 5 shows its basic interface "behind" to some data generated by the functionality AdHoc Report (see below).

- Ad Hoc Report

It aims to provide detailed information on specific areas of performance for consistent decision-making. Two types of ad hoc reports have been implemented, based on the Business Intelligence paradigm: 
- Diagnostic report: provides details on a specific perspective of the SC. The focus of the requested information may be on end-product, business process, SC Member related on products, sales order and shipping order (Figure 5);

- Position paper: used when a current or anticipated problem is identified. The alternative courses of actions (like rescheduling) and probable consequences are drawn up by decisional protocols modeled for each principal producer.

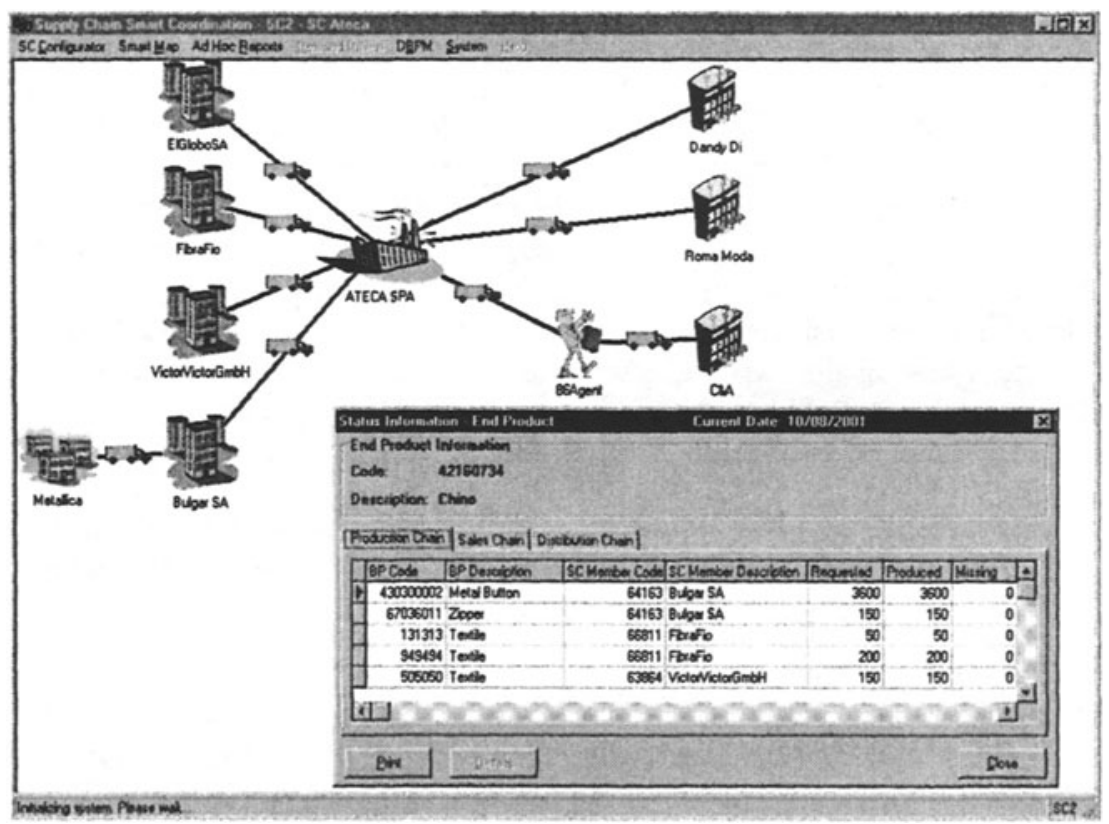

Figure 5 - Ad Hoc Report

- Reactive Inventory

It basically aims to coordinate inventory requirements across multiple distribution centers. The replenishment shipments are initiated when available warehouse stock levels fall below a predetermined minimum or order point.

\section{- Demand Driven}

The distribution \& sales chains are an independent environment where uncertain customer demand determines independent inventory requirements. This functionality allows a faster reaction to conflicts identified in a DBP inventory.

- Distributed Business Process Management

It is a decision-support module that helps the user in solving problems from the production perspective. By means of an intensive information gathering from the SC, a permanent follow up of orders is made as well a set of alternatives schedules are suggested in the presence of problems in the SC schedule.

\subsection{Implementation Aspects}

$\mathrm{SC}^{2}$ has been developed in a PC / Windows-NT / C++ platform. Free software and standards have been used as much as possible, regarding systems efficiency, configurability and interoperability. In terms of CORBA, the C++ ORB TAO (TAO) has been chosen. The system has been modeled in $U M L$, and the multi-agent 
infrastructure has used the MASSYVE KIT (MASSYVE). The (shareware) Interbase database has been utilized, with a simple object-oriented layer to provide the agents to have access to the database as objects and not as table structures.

\section{CONCLUSIONS}

This paper presented the $\mathrm{SC}^{2}$ (Supply Chain Smart Coordination) system, a multiagent-based decision support system that offers some support for agile decisionmaking for management of dynamic supply chains.

This work has been developed in the scope of the IST DAMASCOS project, whose ultimate goal is to develop open and low cost platform and management services for SMEs involved in dynamic supply chains of fashion industries. The functionalities showed were based on the new trends on supply chain management, on an extensive research of related projects, and on the end-users' requirements.

The $\mathrm{SC}^{2}$ system is user-friendly, highly configurable, and quite generic. The architecture has considered real constraints and data, where different strategies of interaction among the existing entities of $\mathrm{SC}^{2}$ had to be conceived, especially for efficiency purposes. Although the level of the agents intelligence is still incipient, it is foreseen a deeper development on this issue in the next future based on the feedback from the project / $\mathrm{SC}^{2}$ end-users.

In the intercontinental tests carried out between the partners' modules, the communication infrastructure, based on CORBA-Workflow-XML, achieved the main goals of being stable, flexible and open.

\subsection{Acknowledgments}

This work has been fully supported by $\mathrm{CNPq}$ - The Brazilian Council for Research and Scientific Development - project n\#480101/00-0. Thanks to André Jacomino, Fabiano Baldo, and Rui Tramontim for the system implementation.

\section{REFERENCES}

1. Camarinha-Matos, L.M, Afsarmanesh, H., The Virtual Enterprise Concept, in Proceedings of the Working Conference on Infrastructures for Virtual Enterprise, pp. 3-14, Porto, Portugal, 1999.

2. Camarinha-Matos, L.M., Afsarmanesh, H.; Rabelo, R.J., Supporting Agility in Virtual Enterprises, Proceedings 2nd IFIP Work. Conf. on Infra. for Virtual Enterprises, pp. 89-104, Brazil, 2000.

3. Damascos, http://bart.inescn.pt/ damascos.

4. Ferreira D., Goletz T., Ferraz, R., A Workflow-Based Approach to the Integration of Enterprise Networks, Proceedings CARS \& FOF 2000, Port of Spain, Trinidad, 2000.

5. Filos, E.; Banahan, E., Will the organization disappear? The challenges of the new economy and future perspectives, Proc. 2nd IFIP Work. Conf. on Infra. for Virtual Enterprises, Brazil, 2000.

6. Jennings, N., Cooperation in Industrial Multi-Agent Systems, World Scientific Series in Computer Science (Vol 43), 1994.

7. Pereira-Klen, A.A.; Rabelo, R. J.; Ferreira, A. C., Supply Chain Smart Coordination in a Virtual Environment, Proc. Int. NAISO Congress on E-Business, Dubai, pp.360-366, 2001(a).

8. Pereira-Klen, A.A.; Rabelo, R. J.; Ferreira, A. C., Spinosa, L.M., Managing DBPs in the Virtual Enterprise, J. Intellig. Manufact., V12 N2, pp. 185-197, ISSN 0956-5515, Kluwer Pub., 2001(b).

9. Pereira-Klen, A.A.; Rabelo, R. J.; Klen, E., D8.1 - System Requirements \& User Needs, internal DAMASCOS Deliverable, Dec 2000.

10. MASSYVE, http://www.gsigma-grucon.ufsc.br/massyve.

11. Rabelo, R. J.; Pereira-Klen, A. A.; Ferreira, A. C., For a Smart Coordination of Distributed Business Processes, Proc. 4th IEEE / IFIP Int. Conf. on IT for Balanced Aut. Sys., Germany, 2000.

12. Rabelo, R. J.; Pereira-Klen, A.A.; Ferreira, A. C., Multi-Perspective Coordination of Supply Chains, Proceedings CARS \& FOF 2001, pp. 484-492, Durban, South Africa, 2001.

13. TAO, http://www.cs.wustl.edu/ schmidt/ACE.html. 